\title{
Blockade of TGF- $\beta$ Signalling Inhibits Cardiac NADPH Oxidase Overactivity in Hypertensive Rats
}

\author{
José Luis Miguel-Carrasco, ${ }^{1}$ Ana Baltanás, ${ }^{1}$ Carolina Cebrián, ${ }^{1}$ María U. Moreno, ${ }^{1}$ \\ Begoña López, ${ }^{1}$ Nerea Hermida, ${ }^{1}$ Arantxa González, ${ }^{1}$ Javier Dotor, ${ }^{2}$ \\ Francisco Borrás-Cuesta, ${ }^{2}$ Javier Díez, ${ }^{1,3}$ Ana Fortuño, ${ }^{1}$ and Guillermo Zalba ${ }^{1}$ \\ ${ }^{1}$ Division of Cardiovascular Sciences, Centre for Applied Medical Research, University of Navarra, 31008 Pamplona, Spain \\ ${ }^{2}$ Division of Hepatology and Gene Therapy, Centre for Applied Medical Research, University of Navarra, 31008 Pamplona, Spain \\ ${ }^{3}$ Department of Cardiology and Cardiac Surgery, University Clinic, University of Navarra, 31008 Pamplona, Spain
}

Correspondence should be addressed to Ana Fortuño, afortuno@unav.es

Received 15 December 2011; Accepted 21 March 2012

Academic Editor: Adrian Manea

Copyright ( 12012 José Luis Miguel-Carrasco et al. This is an open access article distributed under the Creative Commons Attribution License, which permits unrestricted use, distribution, and reproduction in any medium, provided the original work is properly cited.

\begin{abstract}
NADPH oxidases constitute a major source of superoxide anion $\left(\cdot \mathrm{O}_{2}{ }^{-}\right)$in hypertension. Several studies suggest an important role of NADPH oxidases in different effects mediated by TGF- $\beta 1$. In this study we show that chronic administration of P144, a peptide synthesized from type III TGF- $\beta_{1}$ receptor, significantly reduced the cardiac NADPH oxidase expression and activity as well as in the nitrotyrosine levels observed in control spontaneously hypertensive rats (V-SHR) to levels similar to control normotensive Wistar Kyoto rats. In addition, P144 was also able to reduce the significant increases in the expression of collagen type I protein and mRNA observed in hearts from V-SHR. In addition, positive correlations between collagen expression, NADPH oxidase activity, and nitrotyrosine levels were found in all animals. Finally, TGF- $\beta$ 1-stimulated Rat- 2 exhibited significant increases in NADPH oxidase activity that was inhibited in the presence of P144. It could be concluded that the blockade of TGF- $\beta 1$ with P144 inhibited cardiac NADPH oxidase in SHR, thus adding new data to elucidate the involvement of this enzyme in the profibrotic actions of TGF- $\beta 1$.
\end{abstract}

\section{Introduction}

Hypertension is associated with multiple functional and structural cardiovascular alterations $[1,2]$. Among others, these alterations are characterized by the progressive accumulation of fibrillar collagen, namely, collagen type I, in the myocardium of animals and humans with arterial hypertension and left ventricular hypertrophy [3]. Although the exact mechanism by which physiological collagen turns into pathological fibrotic tissue is still unknown, there are many studies that suggest an important role of the local production of the transforming growth factor $\beta 1$ (TGF- $\beta 1$ ) [4]. TGF- $\beta 1$ acts as a key fibrogenic cytokine in many tissues by enhancing extracellular matrix synthesis [5].

Currently, in vitro studies have described that the activation of the nicotinamide adenine dinucleotide phosphate $(\mathrm{NADPH})$ oxidase system has a role in TGF- $\beta$ 1-induced effects [6-9]. Moreover, the association of the NADPH oxidase with TGF- $\beta 1$-induced fibrosis has been also observed in several experimental models [10-13]. The NADPH oxidase has been shown as a major source of superoxide anion $\left(\cdot \mathrm{O}_{2}{ }^{-}\right)$[14]. It consists of a membrane bound cytochrome formed by a small subunit (p22phox) and a big (NOX1-5, Duox1-2) subunit, and in some cases, cytoplasmic subunits that upon phosphorylation bind to the cytochrome [15]. In the heart of rats, Nox2 and Nox4 NADPH isoforms are expressed. There is evidence that the Nox2-dependent form of the enzyme is inducible and generates $\cdot \mathrm{O}_{2}^{-}$, especially by humoral activation $[15,16]$. The Nox4-dependent enzyme seems to be constitutively active and may directly generate hydrogen peroxide $\left(\mathrm{H}_{2} \mathrm{O}_{2}\right)[17,18]$.

It has been described that the synthetic peptide P144, encompassing amino acids 730-743 from the human membrane-proximal ligand-binding domain of TGF- $\beta 1$ 
type III receptor, also called betaglycan, acts as a competitor of TGF- $\beta 1$ type III receptor, sequestering TGF- $\beta$ 1. P144 is able to inhibit fibrosis in a rat model of hepatic failure as well as in a murine model of sclerodermia $[19,20]$. Furthermore, our group has recently demonstrated that P144 prevents myocardial fibrosis and collagen type I synthesis in experimental hypertension [21].

The possible interrelationship between TGF- $\beta 1$ and the NADPH oxidase in cardiac damage has not yet been studied in an experimental model of hypertension. Given that TGF$\beta 1$ is a major contributor to the development of structural alterations in target organs of hypertension [22], we investigated whether the chronic treatment with P144 inhibits cardiac NADPH oxidase and whether this effect is associated with the cardiac antifibrotic properties of the peptide.

\section{Material and Methods}

2.1. Animals. The study was in agreement with the Guide for the Care and Use of Laboratory Animals published by the US National Institutes of Health (NIH Publication no. 85-23, revised 1996) [23], and was approved by the Ethical Committee for Animal Experimentation of the University of Navarra $(090 / 05 ; 100 / 07)$. Rats were provided by Harlan UK Limited (Bicester, UK). Ten-week-old male Wistar-kyoto rats (WKY) $(n=10, \mathrm{~V}-\mathrm{WKY})$ and 10-week-old male spontaneously hypertensive rats (SHR) $(n=10, \mathrm{~V}$-SHR) received vehicle (saline solution) intraperitoneally for 12 weeks, and then were sacrificed at the age of 22 weeks. In addition, 10week-old WKY ( $n=10$, P144-WKY) and 10-week-old SHR $(n=10$, P144-SHR) were treated with intraperitoneal P144 for 12 weeks and then sacrificed. The peptide was dissolved in saline solution, and the concentration was adjusted for the body weight to obtain an average daily dose of $1 \mathrm{mg} / \mathrm{kg}$ body weight/day. This dose was selected because it had been demonstrated previously in rodents that P144 exhibits hepatic and cutaneous antifibrotic activity at doses above $0.5 \mathrm{mg} / \mathrm{kg}$ body weight/day $[15,16]$. All rats were housed in individual cages with free access to standard rat chow and tap water in a quiet room with constant temperature $\left(20-22^{\circ} \mathrm{C}\right)$ and humidity $(50-60 \%)$. Before they were sacrificed by decapitation, the rats were weighed and anaesthetized with Ketamine $75 \mathrm{mg} / \mathrm{kg}$ (Imalgene 1000, Merial) and Xylazine $5 \mathrm{mg} / \mathrm{kg}$ (Rompun, Bayer).

2.2. Measurement of Blood Pressure. Systolic blood pressure (SBP) and diastolic blood pressure (DBP) were measured in all rats every 2 weeks by the standard tail-cuff method using an LE5007 Pressure Computer (Letica Scientific Instruments).

2.3. Preparation of Tissue Samples. After sacrifice, hearts were carefully excised and frozen at $-80^{\circ} \mathrm{C}$ for mRNA, enzymatic activity, and protein analysis.

For NADPH oxidase activity and Western blot studies, hearts were homogenated on ice in phosphate buffer saline (50 $\mathrm{mM} \mathrm{K}_{2} \mathrm{HPO}_{4}, 50 \mathrm{mM} \mathrm{KH}_{2} \mathrm{PO}_{4}, 0.001 \mathrm{mM}$ EDTA, and proteases inhibitor $\mathrm{pH}=7$ ) with a glass/glass motordriven tissue homogenizer for 2 minutes. The homogenate was centrifuged at $2000 \mathrm{~g}$ for 10 minutes. The pellet was discarded, and the supernatant was stored at $-80^{\circ} \mathrm{C}$. Protein concentration was determined by the Lowry methodology.

2.4. NADPH Oxidase Activity. Chemiluminescence assays with $10 \mu \mathrm{mol} / \mathrm{L}$ lucigenin and $200 \mu \mathrm{mol} / \mathrm{L} \mathrm{NADPH}$ were used to measure $\cdot \mathrm{O}_{2}{ }^{-}$production in $200 \mu \mathrm{g}$ of tissue homogenate. Luminescence was recorded during 10 minutes in a tube luminometer (Berthold Detection System, Sirius). A buffer blank was subtracted from each reading, and the value of the area under the curve was used to quantify chemiluminescence. Data are expressed as relative light units (RLUs) produced per second per mg of protein.

2.5. Western Blot. To analyze the nitrotyrosine (NT) levels and the expression of collagen type I and Nox4 and Nox2 NADPH oxidase isoforms, proteins were separated by electrophoresis. Membranes were incubated with specific antibodies against Nox4 and Nox2 isoforms (Santa Cruz Biotechnology, INC Santa Cruz), NT (Upstate Biotechnology, Millipore), and collagen type I (Biogenesis) overnight at $4^{\circ} \mathrm{C}$ at a dilution of $1: 1000$. The membranes were incubated with appropiated peroxidase-conjugated secondary antibodies (GE Healthcare) for 1 hour at room temperature (1:25000 anti-rabbit for Nox4, 1:10000 anti-mouse for Nox2, $1: 10000$ anti-mouse for collagen type I, and $1: 20000$ anti-mouse for NT). Protein expression was visualized with the ECL-Advanced chemiluminescence system (Amersham Biosciences). Bands were analyzed using the Chemidoc Detection System and the Quantity One software (Bio-Rad) obtaining densitometric arbitrary units (AU). The blots were reprobed with a monoclonal $\alpha$-tubulin antibody (Santa Cruz Biotechnology, INC Santa Cruz) as a control for loading. Data are expressed as the relative expression to $\alpha$-tubulin.

2.6. Real-Time RT-PCR. The messenger RNA (mRNA) was isolated from total RNA with the Oligotex mRNA KIT (Quiagen). A real-time PCR reaction was performed using TaqMan Gene Expression probes: Nox4 (Rn 00585380), Nox2 (Rn 00576710), p22phox (Rn 00577357), p47phox (Rn 00586945), TGF- $\beta 1$ (Rn 00572010), fibronectin (Rn 00569575), type I collagen (Rn01463848), biglycan ( Rn 01529736), connective tissue growth factor (CTGF, Rn 00573960), lysyl oxidase (LOX, Rn 01491829), and $18 \mathrm{~S}$ ribosomal RNA(Hs 03003631). Data were normalized to $18 \mathrm{~S}$ ribosomal RNA expression. Relative expression of mRNA was determined by the $2^{-\Delta \Delta \mathrm{CT}}$ method [24].

2.7. Cell Cultures and In Vitro Experiments. The Rat-2 fibroblast cell line was purchased from American Type Culture Collection and maintained in Dulbecco's modified Eagle's medium (Invitrogen) supplemented with 10\% fetal bovine serum (FBS, Invitrogen), Penicilin-Streptomicin solution (Invitrogen), and Fungizone (Invitrogen). Rat-2 cells were seeded at a density of $5.25 \times 10^{5}$ cells in T-25 tissue culture flasks (Nunc) and allowed to adhere overnight. Cells 
were then incubated in reduced-serum medium (1\% FBS) for $24 \mathrm{~h}$ prior to TGF- $\beta 1$ stimulation. Four conditions were used: (i) cells incubated in reduced serum media, (ii) cells incubated in reduced serum media containing TGF$\beta 1(10 \mathrm{ng} / \mathrm{mL})$, (iii) cells incubated in reduced serum media containing P144 $(200 \mu \mathrm{g} / \mathrm{mL})$, and (iv) cells incubated in reduced serum media containing TGF- $\beta 1(10 \mathrm{ng} / \mathrm{mL})$ and P144 $(200 \mu \mathrm{g} / \mathrm{mL})$, for $24 \mathrm{~h}$ prior to harvesting. After treatment, cells were trypsinized and pelleted. The cell pellets were washed in phosphate-buffered solution (Invitrogen) and centrifuged at $1100 \mathrm{~g}$ for $5 \mathrm{~min}$ at $4^{\circ} \mathrm{C}$ and then used for protein extraction.

NADPH oxidase activity was performed as above. In this case, $\cdot \mathrm{O}_{2}{ }^{-}$production was measured on $10 \mu \mathrm{g}$ of cell protein.

2.8. Statistical Analysis. Results are given as mean \pm SEM. To analyze the differences among the four groups, a oneway ANOVA followed by a Scheffe's test was performed once normality had been proven (Shapiro-Wilks test); otherwise, a nonparametric test (Kruskall-Wallis) followed by a MannWhitney $U$-test (adjusting the alpha-level by Bonferroni inequality) was used. Bivariate associations were performed by Pearson's correlation test. The statistical analysis was carried out using the computer program SPSS for Windows version 15.0.

\section{Results}

3.1. Effects of P144 on Blood Pressure. Values of SBP and DBP were elevated in V-SHR (SBP: $246.7 \pm 3.6 \mathrm{mmHg}$; DBP: $208.6 \pm 5.9 \mathrm{mmHg}$ ) at the age of 22 weeks compared with V-WKY (SBP: $175.0 \pm 3.5 \mathrm{mmHg}$; DBP: $128.7 \pm 7.1 \mathrm{mmHg}$; $P<0.01)$. Interestingly, at the age of 22 weeks, blood pressure values were lower $(P<0.01)$ in P144-SHR (SBP: 215.6 $\pm 5.9 \mathrm{mmHg}$; DBP: $191.9 \pm 4.5 \mathrm{mmHg}$ ) than in V-SHR, although they were still higher $(P<0.01)$ than in V-WKY. No significant differences were observed between P144-WKY (SBP: $160.8 \pm 5.2 \mathrm{mmHg}$; DBP: $126.9 \pm 4.3 \mathrm{mmHg}$ ) and VWKY.

3.2. Effects of P144 on Cardiac NADPH Oxidase. Cardiac NADPH oxidase activity was higher $(P<0.05)$ in V-SHR than in V-WKY (Figure 1). The administration of P144 reduced $(P<0.05)$ NADPH oxidase activity in P144-SHR to the V-WKY levels. No differences were found between P144WKY and V-WKY (Figure 1). Accordingly, cardiac mRNA and protein levels of Nox2 (Figure 2) were higher $(P<$ 0.05) in V-SHR than in V-WKY. Compared with V-SHR, the administration of $\mathrm{P} 144$ reduced $\mathrm{mRNA}$ and protein levels of Nox2 (Figure 2) in P144-SHR $(P<0.01)$ to levels similar to V-WKY. Other NADPH oxidase subunits associated with Nox2 NADPH oxidase isoform were also evaluated at mRNA level. Cardiac abundance of p22phox and p47phox was higher $(P<0.01)$ in V-SHR $(2.26 \pm 0.15 \mathrm{AU}$ and $2.23 \pm$ $0.21 \mathrm{AU}$, respectively) than in $\mathrm{V}-\mathrm{WKY}(1.00 \pm 0.09 \mathrm{AU}$ for p22phox and $1.00 \pm 0.08 \mathrm{AU}$ for p47phox). In the same line as the NADPH oxidase activity results, the administration of P144 reduced $(P<0.01)$ the mRNA expression of p22phox

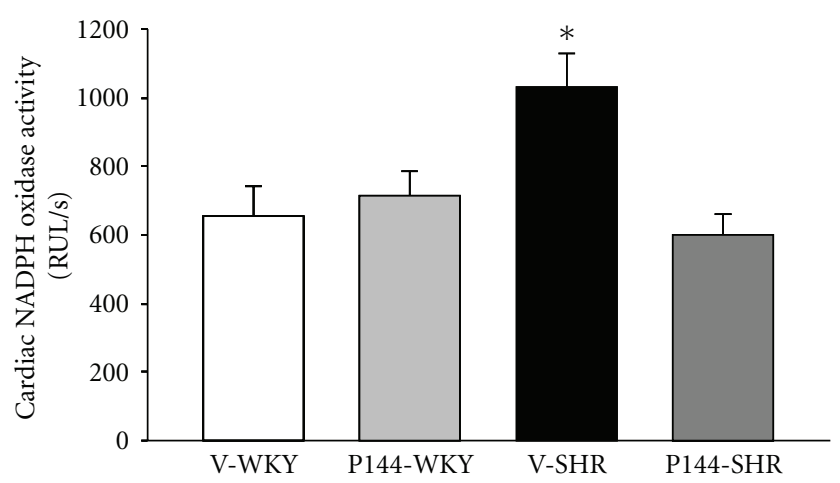

FIgURE 1: Effect of P144 on cardiac NADPH oxidase activity. Cardiac NADPH oxidase-dependent $\cdot \mathrm{O}_{2}{ }^{-}$production was determined by chemiluminescence in the presence of NADPH $200 \mu \mathrm{mol} / \mathrm{L}$, and it is expressed as relative light units per second (RLU/s). Histogram represents mean \pm SEM $(n=10)$. V-WKY means vehicle-treated normotensive rat; P144-WKY, P144-treated WKY rat; V-SHR, vehicle-treated spontaneously hypertensive rat, P144SHR, P144-treated SHR. ${ }^{*} P<0.05$ versus the other groups.

and p47phox on P144-SHR group ( $1.47 \pm 0.1 \mathrm{AU}$ and $0.99 \pm$ 0.23 AU, respectively) to levels similar to V-WKY.

Interestingly, cardiac mRNA and protein levels of Nox4 NADPH oxidase isoform (Figure 3) were higher $(P<$ 0.05) in V-SHR than in V-WKY. Compared with V-SHR, the administration of P144 reduced expression of Nox4 (Figure 3) in P144-SHR $(P<0.01)$ to levels similar to $\mathrm{V}$ WKY.

3.3. Effects of P144 on Cardiac Levels of Nitrotyrosine. Since nitrosylation of proteins is considered a consequence of oxidative stress, the levels of nitrotyrosilated proteins were quantified by Western blot. Following the same pattern that the NADPH oxidase activity determined in heart homogenates, the NT levels were higher $(P<0.05)$ in the V-SHR group than in V-WKY (Figure 4(a)). Similarly, the administration of $\mathrm{P} 144$ reduced $(P<0.05)$ the levels of NT expression in P144-SHR. The levels of nitrotyrosilated proteins were similar between V-WKY and P144-SHR.

A direct correlation was found between the cardiac NADPH oxidase activity and NT levels in all the rats $(r=$ $0.407, P<0.01$ ), suggesting that the NADPH oxidase overactivity may play an important role in the development of cardiac oxidative stress in this model of hypertension (Figure 4(b)).

3.4. Effects of P144 on Cardiac Collagen Metabolism. Cardiac collagen type I protein expression and mRNA levels were higher $(P<0.05)$ in V-SHR than in V-WKY (Figure 5). The administration of $\mathrm{P} 144$ reduced cardiac collagen type I protein expression in P144-SHR. No differences were found between P144-WKY and V-WKY (Figure 5).

The mRNA expression of TGF- $\beta 1$, CTGF, fibronectin, biglycan and LOX was higher $(P<0.01)$ in V-SHR than in $\mathrm{V}$-WKY. The chronic administration of P144 resulted in a decreased expression $(P<0.05)$ of the mRNA levels of these molecules (Figure 6). 


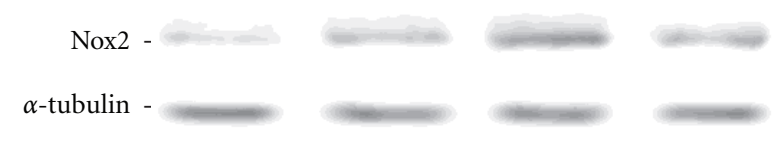

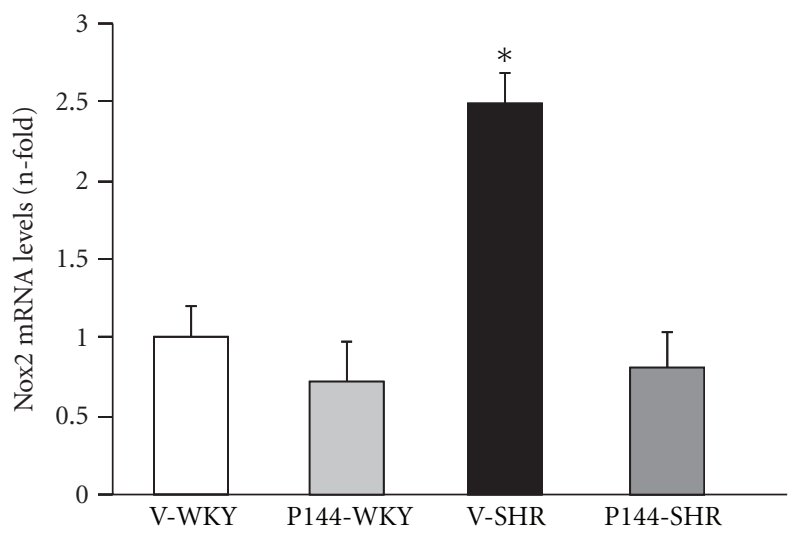

(a)

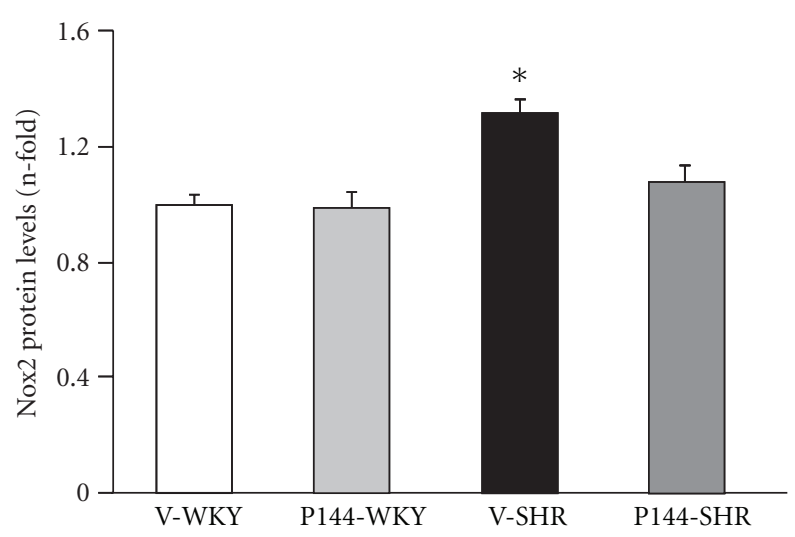

(b)

FIgURE 2: Effect of P144 on cardiac Nox2 subunit expression. (a) mRNA levels of Nox2 expressed as a ratio of gene to $18 \mathrm{~S}$ values from rat heart. (b) Expression of the Nox2 protein levels relative to $\alpha$-tubulin in the rat heart. Representative western blot of the four groups of rats are shown at the upper part. Histograms represent mean \pm SEM $(n=10)$. V-WKY means vehicle-treated normotensive rat; P144-WKY, P144-treated WKY rat; V-SHR, vehicle-treated spontaneously hypertensive rat; P144-SHR, P144-treated SHR. ${ }^{*} P<0.05$ versus the other groups.

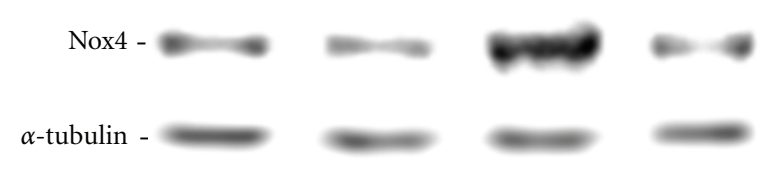

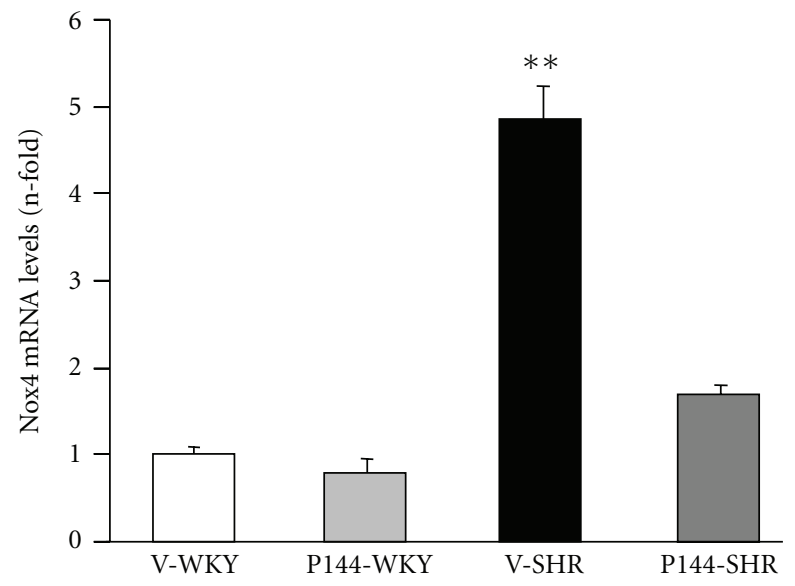

(a)

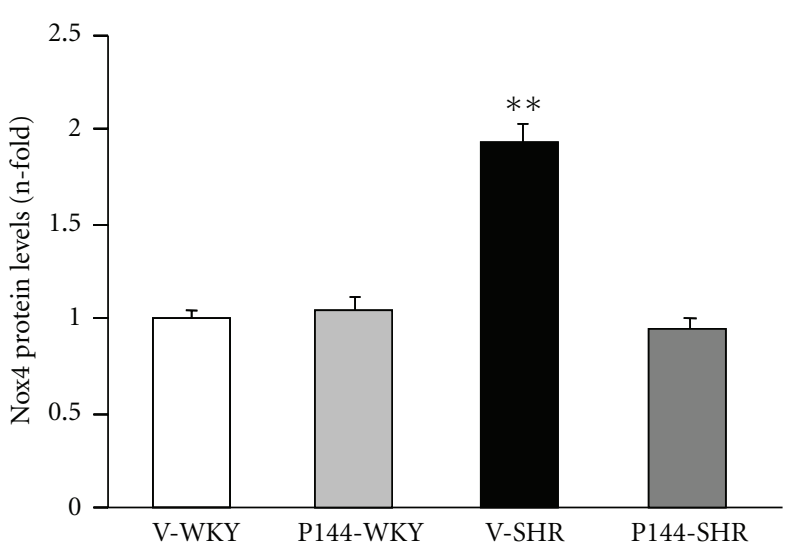

(b)

Figure 3: Effect of P144 on cardiac Nox4 subunit expression. (a) mRNA levels of Nox4 expressed as a ratio of gene to $18 \mathrm{~S}$ values from rat heart. (b) Expression of Nox4 protein levels relative to $\alpha$-tubulin in the rat heart. Representative western blot of the four groups of rats is shown at the upper part. Histograms represent mean \pm SEM $(n=10)$. V-WKY means vehicle-treated normotensive rat; P144-WKY, P144-treated WKY rat; V-SHR, vehicle-treated spontaneously hypertensive rat; P144-SHR, P144-treated SHR. ${ }^{* *} P<0.01$ versus the other groups.

Moreover, combining data from all groups, direct positive correlations were found between collagen type I protein expression and parameters assessing oxidative stress, that is, NT levels $(r=0.384, P<0.01)$ and NADPH oxidase activity $(r=0.372, P<0.05)$.
3.5. In Vitro Findings. In vitro studies showed that TGF- $\beta$ 1 stimulated $(P<0.05)$ the NADPH oxidase activity in Rat-2 fibroblasts (Figure 7). Interestingly, P144 completely prevented the TGF- $\beta 1$-induced NADPH oxidase activity (Figure 7). 

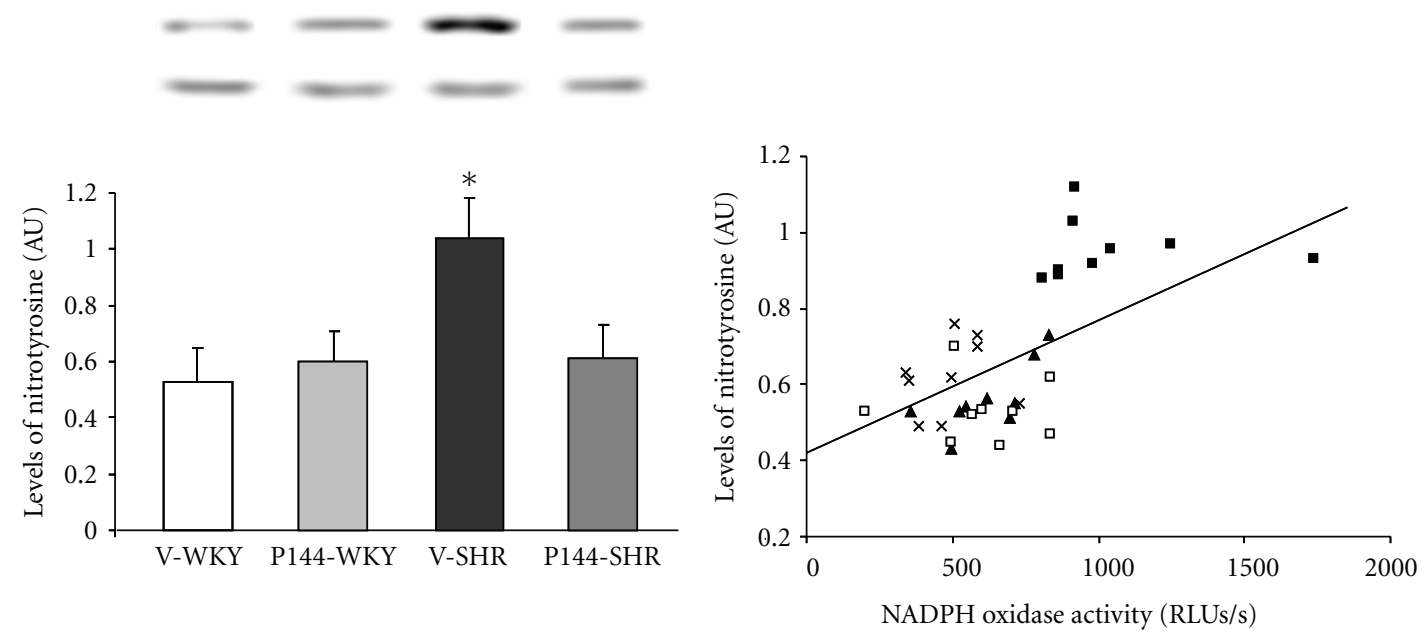

- V-WKY

口 P144-WKY
- V-SHR

$\times$ P144-SHR

(a)

(b)

FIGURE 4: Effect of P144 on cardiac levels of nitrotyrosine (NT). (a) Levels of NT relative to $\alpha$-tubulin in the rat heart. Representative western blot of the four groups of rats are shown at the upper part. Histogram represents mean \pm SEM $(n=10)$. (b) Positive correlation of cardiac NADPH oxidase activity and levels of NT in all the rats $(r=0.407 ; P=0.001 ; y=0.0004 x+0.3797)$. RLU/s means relative light units per second. V-WKY means vehicle-treated normotensive rat; P144-WKY, P144-treated WKY rat; V-SHR, vehicle-treated spontaneously hypertensive rat; P144-SHR, P144-treated SHR. ${ }^{*} P<0.05$ versus the other groups.

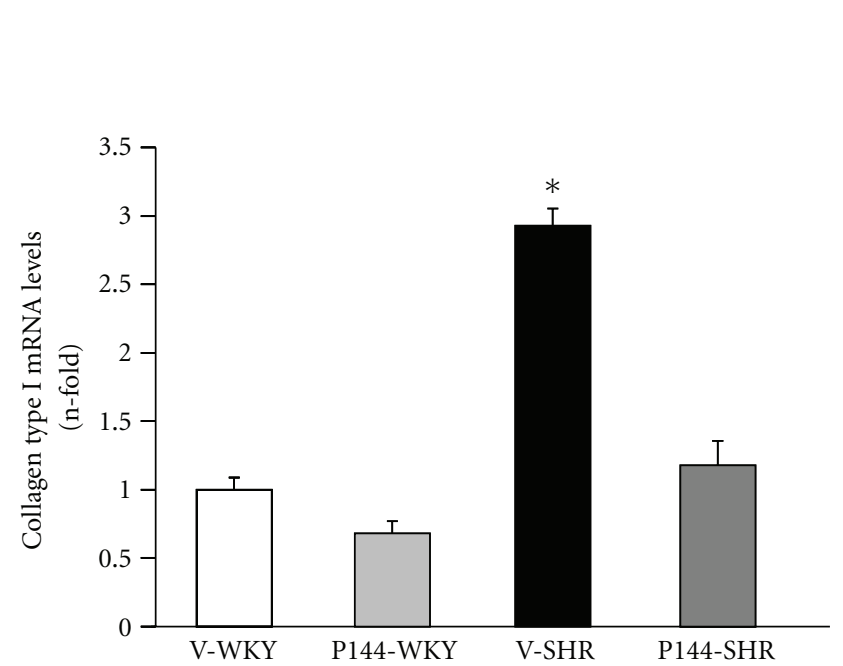

(a)

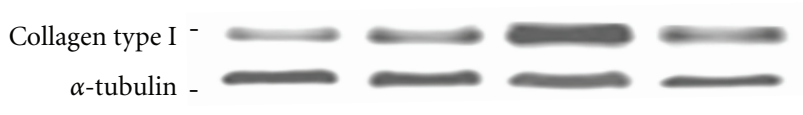

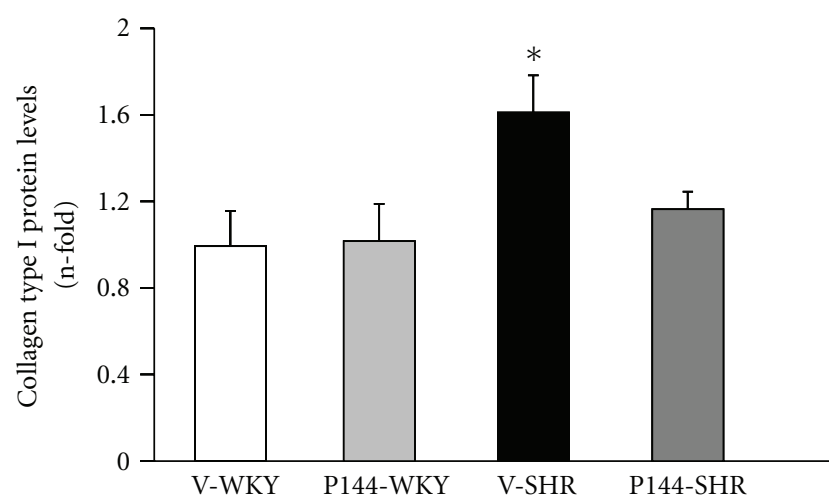

(b)

FIGURE 5: Effect of P144 on cardiac collagen type I expression. (a) mRNA levels of collagen type I expressed as a ratio of gene to $18 \mathrm{~S}$ values from rat heart. (b) Expression of the collagen type I protein relative to $\alpha$-tubulin in the rat heart. Representative western blot of the four groups of rats are shown at the upper part. Histograms represent mean \pm SEM $(n=10)$. V-WKY means vehicle-treated normotensive rat; P144-WKY, P144-treated WKY rat; V-SHR, vehicle-treated spontaneously hypertensive rat; P144-SHR, P144-treated SHR. * P $<0.05$ and ${ }^{*} P<0.05$ versus the other groups.

\section{Discussion}

The main findings of this study are as follows: (i) the chronic administration of P144 inhibits NADPH oxidase activity and expression, as well as NT-assessed oxidative stress in the heart of SHR; (ii) the chronic administration of P144 prevents the excessive expression of cardiac collagen type I and molecules related to collagen synthesis in the same rats; (iii) P144 is able to block the TGF- $\beta$ 1-induced NADPH oxidase activity in cultured rat fibroblasts. Collectively, these findings suggest 


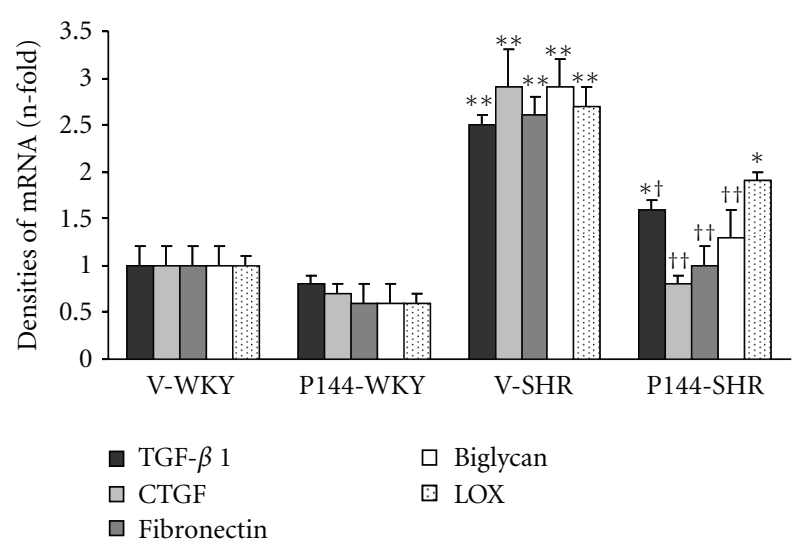

Figure 6: Cardiac mRNA levels of genes assessing collagen metabolism. Cardiac mRNA expression of TGF- $\beta$ 1, connective tissue growth factor (CTGF), fibronectin, biglycan, and lysyl oxidase (LOX) as a ratio of gene to $18 \mathrm{~S}$ values. Histogram represents mean \pm SEM $(n=10)$. V-WKY means vehicle-treated normotensive rat; P144-WKY, P144-treated WKY rat; V-SHR, vehicle-treated spontaneously hypertensive rat; P144-SHR, P144-treated SHR. ${ }^{*} P<0.05$ and ${ }^{*} P<0.01$ versus $\mathrm{V}-\mathrm{WKY} ;{ }^{\dagger} P<0.05$ and ${ }^{+\dagger} P<0.01$ versus $\mathrm{V}-\mathrm{SHR}$.

that interference with the TGF- $\beta$ 1-NADPH oxidase axis by P144 may contribute to the cardiac antifibrotic action of this peptide.

Accumulating evidence indicates that NADPH oxidase activity is increased in several animal models of heart disease $[25,26]$. In our study, increased NADPH oxidase activity in V-SHR associated with upregulated Nox2 and associated subunits, namely, p22phox and p47phox. In addition, the elevation of cardiac NT levels in untreated V-SHR was associated with a parallel increase in NADPH oxidase activity, indicating an increase in oxidative stress in the heart of V-SHR, presumably by NADPH oxidase activation. Interestingly, an increased expression of Nox4 isoform was also observed in the heart of V-SHR. Given that it has been demonstrated that Nox4 NADPH oxidase isoform generates mainly $\mathrm{H}_{2} \mathrm{O}_{2}$ [17], we can speculate that increased cardiac NADPH oxidase activity on V-SHR was due to upregulation of Nox2 isoform and its cytosolic components.

The TGF- $\beta 1$ type III receptor or betaglycan is the most abundant TGF- $\beta 1$ binding protein at the cell surface [27]. Betaglycan potentiates TGF- $\beta 1$ binding to the signaling type I and type II receptors and, therefore, may be involved in ligand presentation [28]. Most of the research to prevent fibrosis in hypertensive heart disease has been focused on the effectiveness of drugs interfering with the angiotensin II-aldosterone-TGF- $\beta 1$ axis $[29,30]$. In this context, our group has developed a synthetic peptide from the sequence of the extracellular region of human betaglycan, the P144, which was predicted as a potential binder to TGF- $\beta 1$ by a computer program [31]. We have recently demonstrated that P144 prevents myocardial fibrosis and collagen type I synthesis in SHR [21].

Diverse experimental approaches have suggested a key role of TGF- $\beta 1$ in the activation of the NADPH oxidase. A study in apolipoprotein E-deficient mice demonstrated that

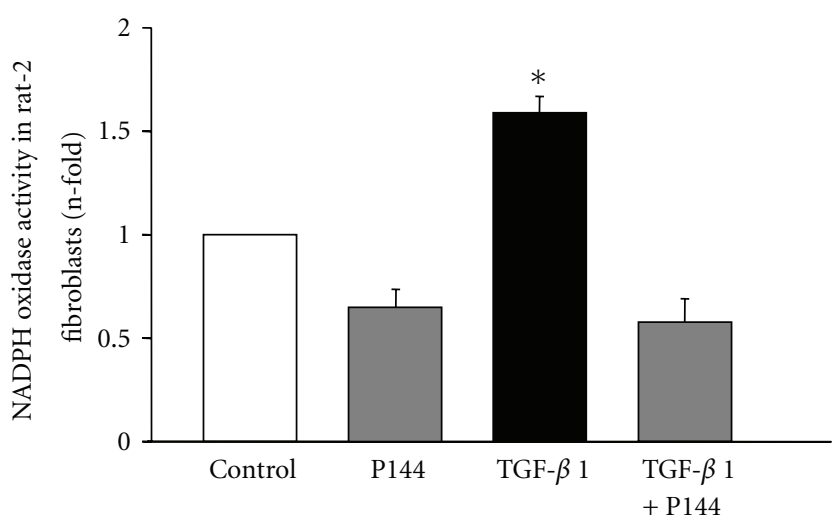

Figure 7: Effect of P144 on TGF- $\beta$-1-induced NADPH oxidase activity in Rat-2 fibroblasts. Cells were incubated with $10 \mathrm{ng} / \mathrm{mL}$ TGF- $\beta 1$ (TGF- $\beta$ 1), $200 \mu \mathrm{g} / \mathrm{mL}$ P144 (P144), and TGF- $\beta 1$ and P144 (TGF- $\beta 1+\mathrm{P} 144)$. Data are expressed as fold increase versus control cells incubated in the absence of TGF- $\beta 1$ and P144 (Control). Histogram represents mean $\pm \operatorname{SEM}(n=10) .{ }^{*} P<0.05$ versus the other groups.

elevated systemic TGF- $\beta 1$ levels cause vascular alterations through the NADPH oxidase activation [32]. In addition, the treatment with benidipine of Dahl salt-sensitive rats with heart failure prevented the cardiac dysfunction and remodeling, which was associated with the suppression of TGF- $\beta 1$ and inhibited the expression of NADPH oxidase subunits [33]. In rat ventricular myocytes, the oxidative stress caused by the NADPH oxidase system mediated the cardiomyocyte contractile dysfunction caused by TGF- $\beta 1$ [34]. In agreement with these studies, we have observed that the treatment with P144 prevented the NADPH oxidase overactivity observed in SHR. In addition, our data showed that the reduction of NADPH oxidase activity by chronic treatment with P144 was associated with a significant reduction of the NADPH oxidase subunit abundance and diminished NT levels in the heart of P144-SHR. Thus, P144 treatment appears to limit oxidative stress by lowering NADPH oxidase abundance in the SHR heart to the levels of the control strain. Studies in adult rat cardiac fibroblasts have shown that NADPH oxidase inhibition blocked the angiotensin II-stimulated collagen production [4]. Finally, the generation of reactive oxygen species in response to Rac has been demonstrated in Rat-2 fibroblasts [35]. Findings from the current study expanded these data by showing that P144 inhibits the ability of TGF- $\beta 1$ to stimulate NADPH oxidase activity in the same cell line of rat fibroblasts.

Several studies have shown that a reduction in NADPH oxidase activation was associated with reversed cardiac damage in several experimental models $[33,36,37]$. In fact, $\mathrm{Wu}$ et al. demonstrated that the antioxidant properties of acetylsalicylic acid were closely associated with its protective effect against angiotensin II-induced cardiac hypertrophy [26]. In addition, the combination of pioglitazone and candesartan exerted beneficial effects on cardiac fibrosis by attenuating the NADPH oxidase activity [37]. The results of the current study allow us to suggest that the antifibrotic 
actions of P144 in experimental hypertension may be associated with its ability to block the TGF- $\beta$-1-induced NADPH oxidase activation. Nevertheless, further experiments should be performed to better address this possibility.

Some limitations of the current study must be recognized. First, the dose of $1 \mathrm{mg} / \mathrm{kg}$ body weight/day was selected to study the effects of P144 on NADPH oxidase-mediated TGF- $\beta 1$ effects. It was previously demonstrated that P144 exhibits hepatic and cutaneous antifibrotic activity at doses above $0.5 \mathrm{mg} / \mathrm{kg}$ body weight per day in rodents [19]. In fact, other groups of SHR and WKY rats were previously treated with P144 at the dose of $0.5 \mathrm{mg} / \mathrm{kg}$ body weight per day, and no antifibrotic effect at cardiac level was observed (data not shown). Second, although we did not determine $\mathrm{H}_{2} \mathrm{O}_{2}$ production, it has been proposed that levels of Nox4 protein expression may be representative of Nox4-dependent $\mathrm{H}_{2} \mathrm{O}_{2}$ production. Finally, to better understand the link between NADPH oxidase and collagen production, further data from in vitro studies would be necessary.

In conclusion, the present results support that P144, a peptide synthesized from type III TGF- $\beta 1$ receptor, prevented NADPH oxidase-dependent oxidative stress and fibrosis in hearts of SHR. Thus, P144 may be interesting as a therapeutic agent for the protection of the heart against oxidative stress and fibrosis in hypertension. Further studies should be performed to better address this possibility.

\section{Abbreviations}

NADPH: Nicotinamide adenine dinucleotide phosphate

TGF- $\beta$ 1: Transforming growth factor- $\beta 1$

$\cdot \mathrm{O}_{2}{ }^{-}$: $\quad$ Superoxide anion

SHR: Spontaneously hypertensive rats

NT: Nitrotyrosine

CTGF: Connective tissue growth factor

LOX: Lysyl oxidase

SBP: $\quad$ Systolic blood pressure

DBP: Diastolic blood pressure.

\section{Acknowledgments}

This paper was funded through the agreement between the Foundation for Applied Medical Research (FIMA) and UTE project CIMA, the Department of Education of Government of Navarra (87/2007), the Ministry of Science and Innovation (RECAVA RD06/0014/0008) (SAF2008-04228), and the European Union (MEDIA project grant HEALTH-F2-2010261409). The authors thank Ana Montoya, Raquel Ros, Idoia Rodríguez, and the Morphology and Imaging Department for their technical support.

\section{References}

[1] M. J. Brown and S. Haydock, "Pathoaetiology, epidemiology and diagnosis of hypertension," Drugs, vol. 59, no. 2, pp. 1-12, 2000.

[2] G. A. Mensah, J. B. Croft, and W. H. Giles, "The heart, kidney, and brain as target organs in hypertension," Current Problems in Cardiology, vol. 28, pp. 156-193, 2003.
[3] J. Díez, A. González, B. López, and R. Querejeta, "Mechanisms of disease: pathologic structural remodeling is more than adaptive hypertrophy in hypertensive heart disease," Nature Clinical Practice Cardiovascular Medicine, vol. 2, no. 4, pp. 209-216, 2005.

[4] P. J. Lijnen, V. V. Petrov, and R. H. Fagard, "Induction of cardiac fibrosis by transforming growth factor- $\beta 1$," Molecular Genetics and Metabolism, vol. 71, no. 1-2, pp. 418-435, 2000.

[5] W. A. Border and N. A. Noble, "Transforming growth factor $\beta$ in tissue fibrosis," New England Journal of Medicine, vol. 331, no. 19, pp. 1286-1292, 1994.

[6] E. Mata-Greenwood, A. Grobe, S. Kumar, Y. Noskina, and S. M. Black, "Cyclic stretch increases VEGF expression in pulmonary arterial smooth muscle cells via TGF- $\beta 1$ and reactive oxygen species: as requirement for $\mathrm{NAD}(\mathrm{P}) \mathrm{H}$ oxidase," American Journal of Physiology, vol. 289, no. 2, pp. L288-L298, 2005.

[7] I. Cucoranu, R. Clempus, A. Dikalova et al., "NAD(P)H oxidase 4 mediates transforming growth factor- $\beta 1$-induced differentiation of cardiac fibroblasts into myofibroblasts," Circulation Research, vol. 97, no. 9, pp. 900-907, 2005.

[8] M. M. Murillo, I. Carmona-Cuenca, G. Del Castillo et al., "Activation of NADPH oxidase by transforming growth factor- $\beta$ in hepatocytes mediates up-regulation of epidermal growth factor receptor ligands through a nuclear factor- $\kappa \mathrm{B}-$ dependent mechanism," Biochemical Journal, vol. 405, no. 2, pp. 251-259, 2007.

[9] V. J. Thannickal and B. L. Fanburg, "Activation of an $\mathrm{H}_{2} \mathrm{O}_{2}$ generating NADH oxidase in human lung fibroblasts by transforming growth factor $\beta 1$," Journal of Biological Chemistry, vol. 270, no. 51, pp. 30334-30338, 1995.

[10] Y. Ishikawa, T. Nishikimi, K. Akimoto, K. Ishimura, H. Ono, and H. Matsuoka, "Long-term administration of Rho-kinase inhibitor ameliorates renal damage in malignant hypertensive rats," Hypertension, vol. 47, no. 6, pp. 1075-1083, 2006.

[11] X. P. Liu, Y. J. Pang, W. W. Zhu et al., "Benazepril, an angiotensin-converting enzyme inhibitor, alleviates renal injury in spontaneously hypertensive rats by inhibiting advanced glycation end-product-mediated pathways," Clinical and Experimental Pharmacology and Physiology, vol. 36, no. 3, pp. 287-296, 2009.

[12] T. Namikoshi, N. Tomita, M. Satoh et al., "Olmesartan ameliorates renovascular injury and oxidative stress in zucker obese rats enhanced by dietary protein," American Journal of Hypertension, vol. 20, no. 10, pp. 1085-1091, 2007.

[13] S. Ohtomo, M. Nangaku, Y. Izuhara, S. Takizawa, C. V. Y. D. Strihou, and T. Miyata, "Cobalt ameliorates renal injury in an obese, hypertensive type 2 diabetes rat model," Nephrology Dialysis Transplantation, vol. 23, no. 4, pp. 1166-1172, 2008.

[14] A. M. Briones and R. M. Touyz, "Oxidative stress and hypertension: current concepts," Current Hypertension Reports, vol. 12, no. 2, pp. 135-142, 2010.

[15] K. Bedard and K. H. Krause, "The NOX family of ROSgenerating NADPH oxidases: physiology and pathophysiology," Physiological Reviews, vol. 87, no. 1, pp. 245-313, 2007.

[16] A. Nabeebaccus, M. Zhang, and A. M. Shah, "NADPH oxidases and cardiac remodelling," Heart Failure Reviews, vol. 16, no. 1, pp. 5-12, 2011.

[17] K. D. Martyn, L. M. Frederick, K. Von Loehneysen, M. C. Dinauer, and U. G. Knaus, "Functional analysis of Nox4 reveals unique characteristics compared to other NADPH oxidases," Cellular Signalling, vol. 18, no. 1, pp. 69-82, 2006.

[18] S. I. Dikalov, A. E. Dikalova, A. T. Bikineyeva, H. H. H. W. Schmidt, D. G. Harrison, and K. K. Griendling, "Distinct roles 
of Nox1 and Nox4 in basal and angiotensin II-stimulated superoxide and hydrogen peroxide production," Free Radical Biology and Medicine, vol. 45, no. 9, pp. 1340-1351, 2008.

[19] B. Santiago, I. Gutiérrez-Cañas, J. Dotor et al., "Topical application of a peptide inhibitor of transforming growth factor$\beta 1$ ameliorates bleomycin-induced skin fibrosis," Journal of Investigative Dermatology, vol. 125, no. 3, pp. 450-455, 2005.

[20] I. J. Ezquerro, J. J. Lasarte, J. Dotor et al., "A synthetic peptide from transforming growth factor $\beta$ type III receptor inhibits liver fibrogenesis in rats with carbon tetrachloride liver injury," Cytokine, vol. 22, no. 1-2, pp. 12-20, 2003.

[21] N. Hermida, B. López, A. González et al., "A synthetic peptide from transforming growth factor- $\beta 1$ type III receptor prevents myocardial fibrosis in spontaneously hypertensive rats," Cardiovascular Research, vol. 81, no. 3, pp. 601-609, 2009.

[22] J. Dabek, A. Kułach, B. Monastyrska-Cup, and Z. Gasior, "Transforming growth factor $\beta$ and cardiovascular diseases: the other facet of the "protective cytokine"'” Pharmacological Reports, vol. 58, no. 6, pp. 799-805, 2006.

[23] US National Institutes of Health, Guide for the Care and Use of Laboratory Animals, NIH Publication no. 85-23, Washington, DC, USA, 1996.

[24] K. J. Livak and T. D. Schmittgen, "Analysis of relative gene expression data using real-time quantitative PCR and the 2$\Delta \Delta$ CT method," Methods, vol. 25, no. 4, pp. 402-408, 2001.

[25] M. C. Wendt, A. Daiber, A. L. Kleschyov et al., "Differential effects of diabetes on the expression of the gp91 phox homologues nox1 and nox4," Free Radical Biology and Medicine, vol. 39, no. 3, pp. 381-391, 2005.

[26] R. Wu, M. A. Laplante, and J. De Champlain, "Prevention of angiotensin II-induced hypertension, cardiovascular hypertrophy and oxidative stress by acetylsalicylic acid in rats," Journal of Hypertension, vol. 22, no. 4, pp. 793-801, 2004.

[27] J. Massagué and B. Like, "Cellular receptors for type $\beta$ transforming growth factor. Ligand binding and affinity labeling in human and rodent cell lines," Journal of Biological Chemistry, vol. 260, no. 5, pp. 2636-2645, 1985.

[28] F. López-Casillas, J. L. Wrana, and J. Massagué, "Betaglycan presents ligand to the TGF $\beta$ signaling receptor," Cell, vol. 73, no. 7, pp. 1435-1444, 1993.

[29] W. Arozal, K. Watanabe, P. T. Veeraveedu et al., "Effects of angiotensin receptor blocker on oxidative stress and cardiorenal function in streptozotocin-induced diabetic rats," Biological and Pharmaceutical Bulletin, vol. 32, no. 8, pp. 1411-1416, 2009.

[30] K. Nagata, F. Somura, K. Obata et al., "AT1 receptor blockade reduces cardiac calcineurin activity in hypertensive rats," Hypertension, vol. 40, no. 2, pp. 168-174, 2002.

[31] J. Esparza-López, J. L. Montiel, M. M. Vilchis-Landeros, T. Okadome, K. Miyazono, and F. López-Casillas, "Ligand binding and functional properties of betaglycan, a co-receptor of the transforming growth factor- $\beta$ superfamily. Specialized binding regions for transforming growth factor- $\beta$ and inhibin A," Journal of Biological Chemistry, vol. 276, no. 18, pp. 1458814596, 2001.

[32] A. Buday, P. Orsy, M. Godó et al., "Elevated systemic TGF$\beta$ impairs aortic vasomotor function through activation of NADPH oxidase-driven superoxide production and leads to hypertension, myocardial remodeling, and increased plaque formation in apoE -/- mice," American Journal of Physiology, vol. 299, no. 2, pp. H386-H395, 2010.

[33] T. Ohno, N. Kobayashi, K. Yoshida, H. Fukushima, and H. Matsuoka, "Cardioprotective effect of benidipine on cardiac performance and remodeling in failing rat hearts," American Journal of Hypertension, vol. 21, no. 2, pp. 224-230, 2008.

[34] S. Li, X. Li, H. Zheng, B. Xie, K. R. Bidasee, and G. J. Rozanski, "Pro-oxidant effect of transforming growth factor- $\beta 1$ mediates contractile dysfunction in rat ventricular myocytes," Cardiovascular Research, vol. 77, no. 1, pp. 107-117, 2008.

[35] C. H. Woo, Z. W. Lee, B. C. Kim, K. S. Ha, and J. H. Kim, "Involvement of cytosolic phospholipase A2, and the subsequent release of arachidonic acid, in signalling by Rac for the generation of intracellular reactive oxygen species in Rat-2 fibroblasts," Biochemical Journal, vol. 348, no. 3, pp. 525-530, 2000.

[36] I. Papparella, G. Ceolotto, L. Berto et al., "Vitamin C prevents zidovudine-induced $\mathrm{NAD}(\mathrm{P}) \mathrm{H}$ oxidase activation and hypertension in the rat," Cardiovascular Research, vol. 73, no. 2, pp. 432-438, 2007.

[37] T. Nakamura, E. Yamamoto, K. Kataoka et al., "Beneficial effects of pioglitazone on hypertensive cardiovascular injury are enhanced by combination with candesartan," Hypertension, vol. 51, no. 2, pp. 296-301, 2008. 


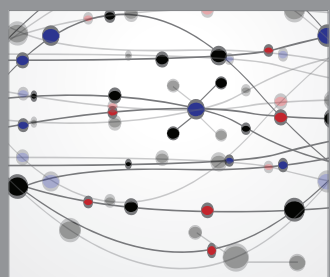

The Scientific World Journal
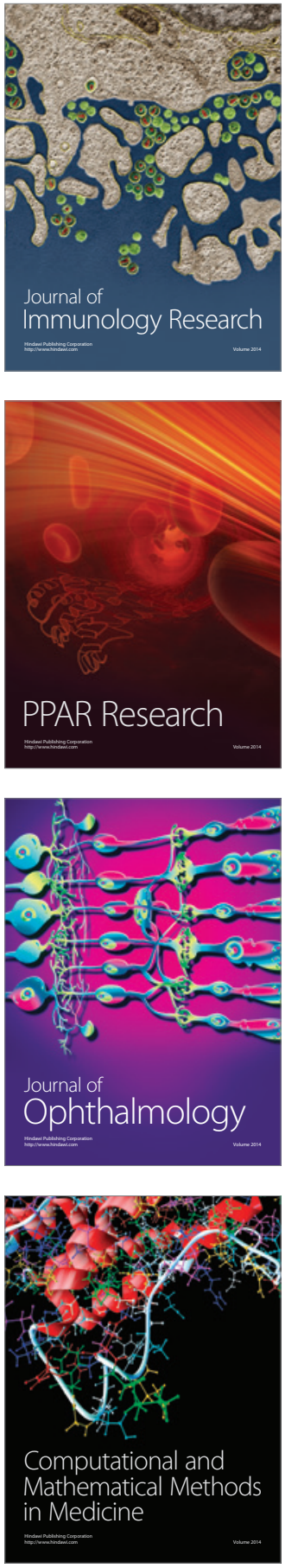

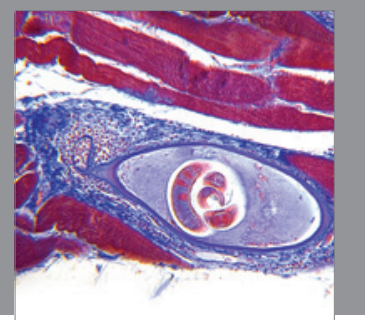

Gastroenterology

Research and Practice
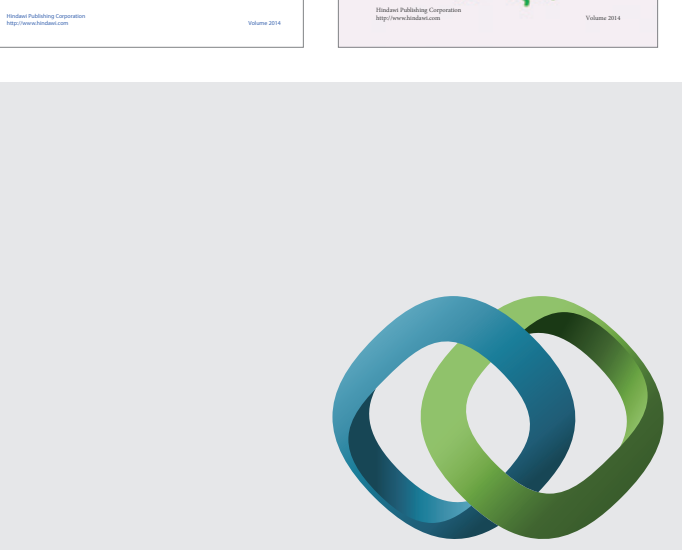

\section{Hindawi}

Submit your manuscripts at

http://www.hindawi.com
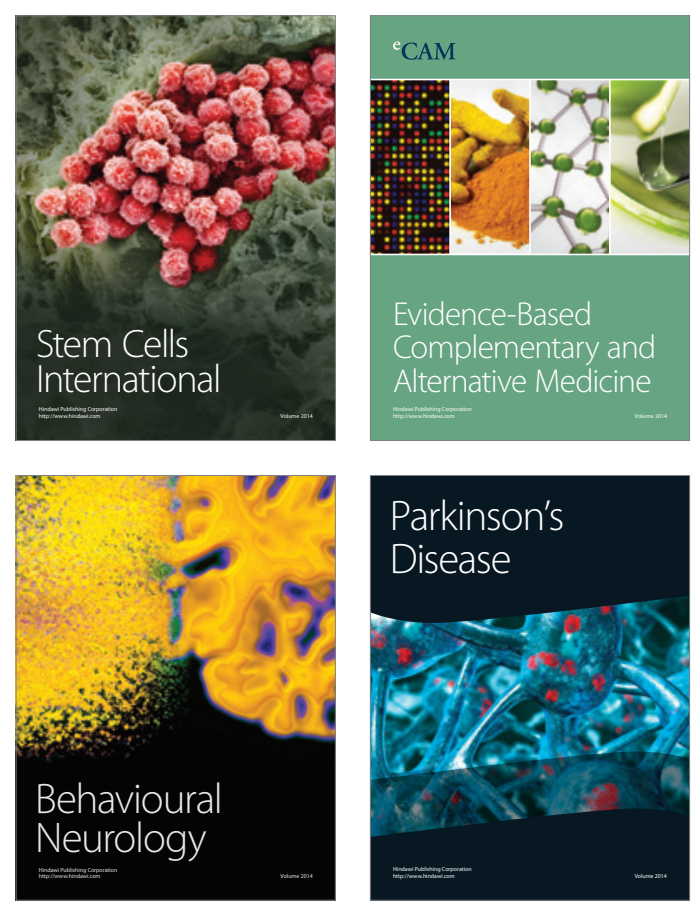

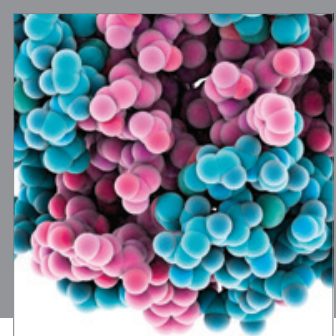

Journal of
Diabetes Research

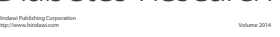

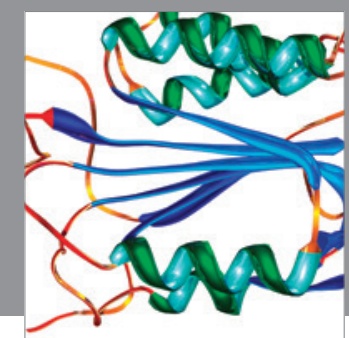

Disease Markers
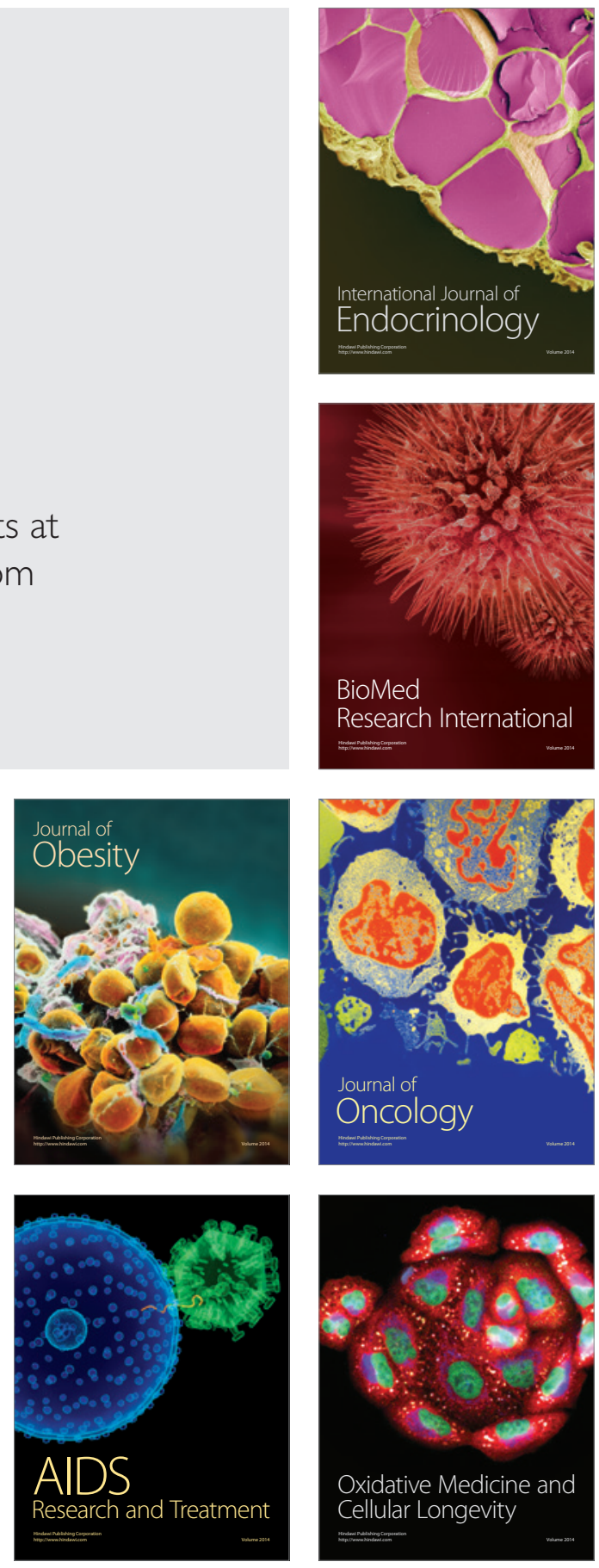